\title{
Virtual Reality Games for Health Care
}

\author{
Alice J Lin ${ }^{1,}$, Charles B Chen ${ }^{2}$ and Fuhua (Frank) Cheng ${ }^{3}$ \\ ${ }^{1}$ Department of Computer Science and Information Technology, Austin Peay State University, Clarksville, TN 37044, USA \\ ${ }^{2}$ Department of Pediatrics, Cleveland Clinic, Cleveland, OH 44195, USA \\ ${ }^{3}$ Department of Computer Science, University of Kentucky, Lexington, KY 40506, USA
}

\begin{abstract}
Virtual reality (VR) and interactive technologies have become increasingly common in today's world, and there are many applications for this technology in numerous fields. In this paper, we present a review on recent and ongoing research related to applications of VR and VR games in the healthcare field. VR technology is allowing medical practitioners to help their patients in a number of innovative ways in addition to helping health care providers improve their skills. Lastly, we propose a VR game design for rehabilitation and palliative care.
\end{abstract}

\section{Introduction of VR Games}

Immersive and interactive technologies such as Virtual Reality (VR), are becoming increasingly more prevalent in our everyday lives. They are a new milestone in the way we interact with our environment, and even how we conceive new approaches in our relationship with reality [1]. VR and other immersive Information and Communication Technologies have a high potential for transforming the real world and the way in which we interact with it [2].

VR or virtual environment (VE) can be defined as a computing technology that generates a simulated or artificially three dimensional (3D) environment, which imitates reality. VR presents a convincing interface that allows the user to engage with the computer-generated environment in a naturalistic way. Through 3D computer graphics via advanced input and output devices, users believe they actually perceive sensory information that is similar to that of the real world. In very simple terms, virtual reality can be defined as a synthetic or virtual environment which gives a person a sense of reality [3].

Games beyond entertainment have been explored in various application domains such as education and training for decades. Typical applications of VR and games technologies in health care include therapy, pain relief [4], surgical procedures, patient education, medical training, skill enhancement and rehabilitation [5], etc. To date, such applications have improved the quality of health care, the quality of life of the patient, and accessibility to health care [3].

\section{Current VR Games for Health care}

When we think about VR, we tend to associate it with the entertainment genre. Although VR indeed set sail to enhance the demanding gamer's experience, it has also made significant improvements in the lives of people with symptoms [6].

According to the Human Interface Technology Lab at the University of Washington [4, 7], VR applications have an analgesic effect and "numb" these areas of the brain, making them less active and helping patients cope with damage better. Floreo is leveraging the power of VR to develop a supplemental method of teaching social and communication skills for individuals with Autism Spectrum Disorder [8]. VisitU provides patients with a downloadable application and virtual glasses which allow them to get in touch with their home and loved ones at any time of the day or night in the participating hospitals [9]. Products like IrisVision help patients with poor vision regain their sight via a VR experience [10]. We Are Alfred is a VR-powered product created by Embodied Labs that aims to let young medical students understand what it is like to live as a 74-year-old man with visual and hearing impairments. Each user can experience life from the older patient's perspective [11]. Mindmaze allows patients to practice how to move their fingers or lift their arms in a fun fashion with the help of VR. Although patients do not carry out the actual movement, their engagement, motivation, and attention is notably improved with audiovisual feedback, which could speed the recovery of traumatized nervous systems [12]. The Johnson \& Johnson Institute has launched a new global VR training program for surgeons and nurses [13].

Shafi Ahmed performed an operation at the Royal London hospital using a VR camera connected to the Medical Realities website. That gave real-time access to all interested parties that wished to participate in the operation, including worried relatives and journalists [14].

The use of VR and commercial gaming systems has received attention for enabling physical activity and engagement in healthy individuals and for rehabilitation in

a Corresponding author: lina@apsu.edu 
clinical populations [15-20]. When coupled with popular, commercially available gaming systems, the engaging nature of these activities can provide a motivating and enjoyable means of adhering to exercise and increasing physical activity in the comfort and convenience of one's own home [21]. The Rehabilitation Gaming System (RGS) is a VR based system which helps patients for neurorehabilitation that is based on a number of hypotheses on the neuronal mechanisms underlying recovery, the structure of training, and the role of individualization [22].

From autism and low vision to chronic pain, VR's immersive technology is proving to be a revolutionary solution to cases where conventional methods fail. Additionally, it provides an affordable and safe way for patients to better their lives and regain the life they have lost due to pain or other health-related issues. With all that in mind, it will not be surprising if VR therapies take center stage in the US healthcare system in the near future [6].

\section{Discussion of Current Applications}

Virtual environments and related technologies add value to healthcare in the areas of cost savings, improved services, and savings in material resources [23]. Virtual reality is greatly impacting and improving health care results [24]. What is the future of virtual reality and immersive 3D environments? The interest of research in this technology lies in the possibility of its use, application and implementation in a wide range of research domains and disciplines.

\section{Our Approach}

We will design and develop an innovative multiplayer VR game. The VR game can be played by more than one person in the same game environment at the same time, either locally or over the Internet. It allows players' interaction with other individuals in partnership, competition or rivalry, providing them with social communication. Players may compete against two (or more) human contestants, work cooperatively with a human partner to achieve a common goal. Players can choose avatars or create their own avatars in virtual world. If the player is in the hospital or home, his/her family, relatives or friends is in halfway across the country, they can visit him/her any time or hang out together in an immersive virtual world. Instead of play game, players can watch the game as a spectator.

Our immersive interactive system includes: interactive computer graphics, immersive environment with head tracking, head mounted display (HMD). Gloves, wands or touch controllers for interaction and these allow players to reach out and feel things that aren't really there. They also allow you to interact with objects. You can pick up an object and do things with it, just like you would with a real object. The built-in microphone makes it easy to communicate with teammates.

Our game is rendered in real-time with Unity 3D and Oculus Rift. Our system is fully wireless. Positions are constantly tracked (head, hands, or whole body), and positions of objects in the environment are constantly updated. The display is redrawn with new view positions, new user body configurations and new object locations.

Motion Tracking Hardware including gyroscopes, accelerometers and other low-cost components are used in VR to sense when our bodies move and our heads turn, so that the application can update our view in the 3D scene. The game will utilize Oculus Rift for VR displaying and Atlas for positioning. By placing markers in a room and using an app and an iPhone (mounted on a player's chest) to track where the player is within that environment, we can convert a real-world space into a virtual space.

These markers may be used as the boundaries for the game area or as the land markers inside the game area. First we find an open space. If there is a big living room it could work, but several connected rooms will be better (Figure 1). The play booth (Figure 2) can be placed in one part of a room. For the other rooms, we will map the real world into a virtual world to set the boundaries of the game space. What this allows is for an Oculus Rift wearer to move around freely. The phone's accelerometer and gyroscopes will pinpoint the exact location.

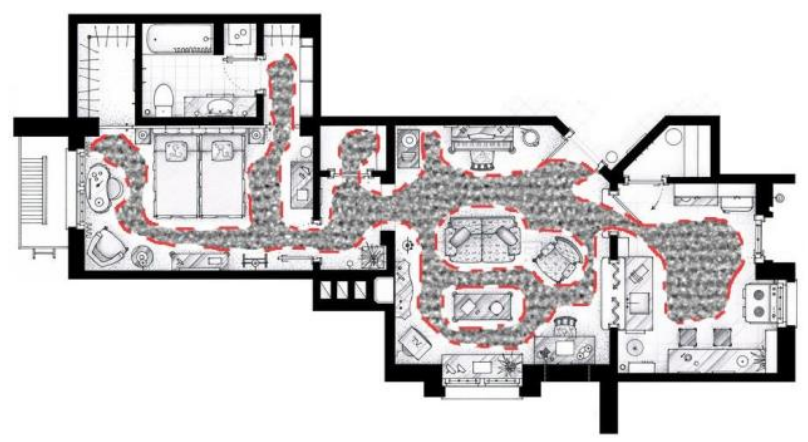

Figure 1. Real space can be mapped into virtual space. This floor map shows connected rooms. The red broken lines indicate the boundaries in virtual space. The carpeted area is the virtual space that the player can freely play on.

Figure 3 shows our overall system which combines VR and gaming fields. We will develop our serious game with Unity as the game engine. Atlas will be used for players to walk, run, jump, crouch, bend and move around like they do in real life. In the VR game interface, there will be a health index meter, main menu, map, level, and score to indicate the game progress and the player's virtual health status. The player's action will be statistically analyzed and player's health index is subsequently determined.

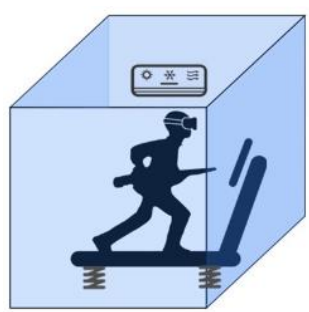

Figure 2. Setting of the play booth: treadmill, air-conditioner, and springs under the treadmill for generating vibration. 


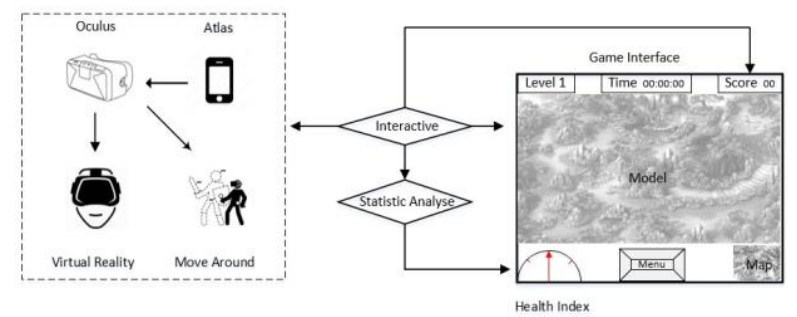

Figure 3. The overall structure of the game

To enhance the VR experience, the play booth will be equipped with an air-conditioner, vibrating equipment, and a treadmill. The air-conditioner is used to blow air of different temperatures to simulate different environments and scenes.

The vibrating equipment will shake a bit when players are in an unstable place such as a pedestrian-only suspension bridge. The function of the equipment in the center can be disabled (Figure 2). Each player has their own dedicated booth/room, specially designed and equipped with the virtual reality gear. They will participate in the game standing up and will get to move around, search for items, manipulate objects and collaborate with their fellow team members.

This is a game where only the player's creative energies determine the user's interaction with the community. Players can create virtual representations of themselves as well as other avatars. The players create virtual representations of themselves and are able to interact with other avatars, places or objects. It is a platform that principally features 3D based user-generated content. Built into the software is a three-dimensional modeling tool based on simple geometric shapes that allows the player to build virtual objects. Other objects, animations, and gestures can be created using external software.

A variety of levels will be built into the game. The player will be able to navigate each sub-system. Each new level provides additional challenges, and when a level is successfully completed, the player will get a new score. Upon completion of all levels, the final score will be displayed. The game will consist of several sections, for different age groups. Each section contains three or four levels. Each level has an immersive VR movie as a tutorial. Whether the players are stepping into the game, watching an immersive VR movie, or jumping to a destination on the other side of the world, they will feel as if they are really there. Players must overcome challenges, gain new levels, and acquire new and more powerful objects if they wish to proceed in the game.

The non-player characters (NPCs) are the heart and soul, and are populated at a given level. NPCs are controlled by the computer and are the main source of conflict during gameplay. NPCs are one of the elements we will develop to craft the pacing, challenge, and tension of a level. We can control not only where the NPCs are placed but also the NPCs' scripted behavior, how they are equipped, their level of health, their armor, and other variables [25]. We will develop artificial intelligence to give nonhuman entities algorithmic and mathematical solutions for problem solving and simulating human thought and decision making.

The player's actions will be incorporated into the computer model and reflected in a way the player can perceive. We will employ passive haptics to augment interaction and perception in the game to enhance the virtual environment. Passive haptics are real objects in a physical space that are mapped to virtual objects in a virtual space. Players wear a HMD while in the physical space. When they look toward the physical object, they will see the virtual representation of it in their display. When they approach the object and try to touch it, they encounter the real object in the physical space. Anything a player does with that object in real space appears as a reflected action upon the virtual object in virtual space.

The interaction between the technology and the player is paramount. Therefore, it is important to assess the player's perceptions or actions to determine whether the VR game has been well designed and employed and whether it provides meaningful feedback and knowledge of results to the individual. We will develop a VR assessment game. It has the capability to precisely recreate situations or environments across different sessions at different times or places. It has the power to record behavior with detail and precision.

\section{Conclusion}

Nowadays, immersive environments and interactive technologies are a research domain with applications in various fields of knowledge, as well as educational approaches and even as a tool for social change [2].

Research has shown that video games have numerous therapeutic benefits for individuals living with chronic diseases [26].

The aim of our VR game is to improve the quality of life of people living with chronic diseases through interactive and educational VR gaming. As many people are limited physically, they often do not have the ability to perform the actions that are demonstrated in regular games (e.g. running, jumping). Allowing them to play and engage in a VR environment that allows them to be seemingly able to perform those actions can be extremely gratifying. As many sick or disabled people are depressed or suffer from other psychological disorders, helping them engage in a different environment (especially one that temporarily takes away their physical limitations) can significantly improve their mood. The player will experience a truly exhilarating and mind-blowing adventure, immersion, freedom, and the ultimate thrill with his or her friends, family, or colleagues as they communicate and work together in a totally realistic virtual world. At the same time, these games can teach specific lessons about managing chronic illnesses and reinforce key messages about maintaining one's own health.

\section{References}

1. A. Kitson, M. Prpa, and B. E. Riecke, "Immersive Interactive Technologies for Positive Change: A 
Scoping Review and Design Considerations," Frontiers in Psychology, vol. 9, pp. 1-19, 2018.

2. J. L. Rubio-Tamayo, M. G. Barrio, and F. G. García, "Immersive Environments and Virtual Reality: Systematic Review and Advances in Communication, Interaction and Simulation," Multimodal Technologies Interact., vol. 1, 2017.

3. M. Ma and H. Zheng, Virtual Reality and Serious Games in Healthcare, Springer, 2011.

4. S. R. Sharar, W. Miller, A. Teeley, M. Soltani, H. G. Hoffman, M. P. Jensen, and D. R. Patterson, "Applications of virtual reality for pain management in burn-injured patients," Expert Rev Neurother., vol. 8, pp. 1667-1674., 2008.

5. M. Ma and K. Bechkoum, "Serious games for movement therapy after stroke," IEEE International Conference on Systems, Man and Cybernetics, vol., 2008.

6. M. Usman, 7 Ways VR is Improving Healthcare, 2018.

7. S. R. Sharar, A. Alamdari, C. Hoffer, H. G. Hoffman, M. P. Jensen, and D. R. Patterson, "Circumplex Model of Affect: A Measure of Pleasure and Arousal During Virtual Reality Distraction Analgesia," GAMES FOR HEALTH JOURNAL, vol. 5, pp. 197-202, 2016.

8. J. Parish-Morris, R. Solórzano, V. Ravindran, V. Sazawal, S. Turnacioglu, A. Zitter, J. Miller, and J. McCleery, Immersive Virtual Reality to Improve Police Interaction Skills in Adolescents and Adults with Autism Spectrum Disorder: Preliminary Results of a Feasibility and Safety Trial, 2018.

9. VisitU, What VisitU does, 2018.

10. IrisVision Global Inc, Breakthrough Wearable Low Vision Aid Restores Life's Limitless Possibilities, 2018.

11. M. Meacham, A New Reality: Learning empathy through Virtual Reality, 2017.

12. Mindmaze, Increase your patient's recovery potential throughout the continuum of care, 2018.

13. The Johnson \& Johnson Institute, Introducing Global Virtual Reality Training for Surgeons and Nurses, 2018.

14. N. Davis, Cutting-edge theatre: world's first virtual reality operation goes live, 2016.

15. B. S. Lange, P. Requejo, S. M. Flynn, A. A. Rizzo, F. J. Valero-Cuevas, L. Baker, and C. Winstein, "The Potential of Virtual Reality and Gaming to Assist
Successful Aging with Disability," Phys Med Rehabil Cli, vol. 21, pp. 339-356, 2010.

16. K. E. Laver, B. Lange, S. George, J. E. Deutsch, G. Saposnik, and M. Crotty, Virtual reality for stroke rehabilitation, 2017.

17. Taylor MJD, McCormick D, Shawis T, Impson R, and Griffin M, "Activity-promoting gaming systems in exercise and rehabilitation," J Rehabil Res Dev, vol. 48, pp. 1171-1186, 2011.

18. Peng W, Crouse JC, and Lin JH, "Using active video games for physical activity promotion: a systematic review of the current state of research," Health Educ Behav., vol. 40, pp. 171-192, 2013.

19. W. Peng, J. C. Crouse, and J.-H. Lin, "Using Active Video Games for Physical Activity Promotion: A Systematic Review of the Current State of Research," Health Education \& Behavior, vol. 40, pp. 171-192, 2012.

20. M. J. D. Taylor, D. McCormick, T. Shawis, R. Impson, and M. Griffin, "Activity-promoting gaming systems in exercise and rehabilitation," Journal of Rehabilitation Research \& Development, vol. 48, pp. 1171-1186, 2011.

21. K. J. Miller, B. S. Adair, A. J. Pearce, C. M. Said, E. Ozanne, and M. M. Morris, "Effectiveness and feasibility of virtual reality and gaming system use at home by older adults for enabling physical activity to improve healthrelated domains: a systematic review," Age and Ageing, vol. 43, pp.: 188-195, 2014.

22. M. S. Cameirão, S. B. i. Badia, E. D. Oller, and P. F. Verschure, "Neurorehabilitation using the virtual reality based Rehabilitation Gaming System: methodology, design, psychometrics, usability and validation," Journal of NeuroEngineering and Rehabilitation, vol. 7, pp. 1-14, 2010.

23. J. Moline, "Virtual Reality for Health Care: a survey," Stud Health Technol Inform, vol. 44, pp. 3-34, 1997.

24. C. Chinnock, "VR Can Improve your Health," Byte, vol. 20, p 26, 1995.

25. G. Rivera, K. Hullett, and J. Whitehead, "Enemy NPC Design Patterns in Shooter Games," Proceedings of the First Workshop on Design Patterns in Games, vol., 2012.

26. The University of Utah, Video Games Help Patients and Health Care Providers, 2016. 